\title{
Does the elevation of CAMLs predict metastatic breast cancer patients' survival?
}

\author{
Qixing Tan ${ }^{1} \cdot$ Qinghong $\left.^{\text {Qin }^{1}}{ }^{(}\right)$
}

Received: 12 October 2017 / Accepted: 16 October 2017 / Published online: 20 October 2017

(C) Springer Science+Business Media, LLC 2017

To the Editor,

With great interest, we read an article entitled "Prognostic values of cancer associated macrophage-like cells (CAML) enumeration in metastatic breast cancer", which was recently written by $\mathrm{Mu}$ et al. and published in Breast Cancer Res Treat [1]. In this article, they evaluated the prognostic significance of CAML in the cases of metastatic breast cancer (MBC). They found that CAMLs is an independent prognostic biomarker at baseline for the patients with MBC. Even though we approve of their application of this biomarker to the challenging prediction of the prognosis of the patients with MBC, we deem that certain significant problems should be solved before their conclusions can be recognized. In our opinion, the first and most important limitation of their study is that their inclusion criteria were indistinct. In the cases of $\mathrm{MBC}$, prognosis strongly depends on age, viscera function, the location and size of metastatic tumor, pathologic types, and treatment history [2]. For instance, the prognoses of bone metastasis and visceral metastasis are obviously different, even though both of them are metastatic breast cancers [3]. Therefore, it is of great importance to judge whether those patients involved in their study were comparable. The authors should analyze other factors that may affect prognosis, and set clear inclusion criteria. Additionally, the blood samples utilized for the counting of CAMLs and circulating tumor cells (CTCs) were obtained from patients as baseline samples before starting a new therapy, whether the initial

Qinghong Qin

qqh108@aliyun.com

1 Department of Breast Surgery, The Affiliated Cancer

Hospital of Guangxi Medical University, 71 He Di Rd.,

Nanning 530021, Guangxi, People's Republic of China treatment would influence the enumerations of these cells is also worth discussing. As a result, their work poses an intriguing question about the prognostic effectiveness of CAMLs in MBC. However, their findings still need more thorough analyses so that they would be acknowledged.

Compliance with ethical standard

Conflict of interest The authors declare that they have no conflict of interest.

Ethical approval This manuscript complies with the ethical rules applicable for this journal.

Human and animal rights This article does not contain any studies with human participants or animals performed by any of the authors.

\section{References}

1. Mu Z, Wang C, Ye Z, Rossi G, Sun C, Li L, Zhu Z, Yang H, Cristofanilli M (2017) Prognostic values of cancer associated macrophage-like cells (CAML) enumeration in metastatic breast cancer. Breast Cancer Res Treat. doi:10.1007/s10549-017-4372-8

2. Jung SY, Rosenzweig M, Sereika SM, Linkov F, Brufsky A, Weissfeld JL (2012) Factors associated with mortality after breast cancer metastasis. Cancer Causes Control: CCC 23(1):103-112. doi:10.1007/s10552-011-9859-8

3. Ording AG, Heide-Jorgensen U, Christiansen CF, Norgaard M, Acquavella J, Sorensen HT (2017) Site of metastasis and breast cancer mortality: a Danish nationwide registry-based cohort study. Clin Exp Metas 34(1):93-101. doi:10.1007/s10585-016-9824-8 